\title{
La validez de un registro clínico de úlceras por presión
}

\section{Validity of clinical register of pressure ulcers}

\author{
T. Sebastián-Viana ${ }^{1}$, J.M. González-Ruiz ${ }^{2}$, F. Núñez-Crespo ${ }^{3}$, I. Lema-Lorenzo², \\ G. Gadea-Romero ${ }^{2}$, M.E. Losa-Iglesias ${ }^{4-*}$
}

\section{RESUMEN}

Objetivos. Comparar las úlceras por presión (UPP) encontradas en este estudio observacional prospectivo con las registradas en la historia clínica electrónica (HCE).

Método. Estudio observacional prospectivo para registrar las UPP, con seguimiento diario por una observadora en las unidades de hospitalización médico-quirúrgicas y revisión del registro clínico de seguimiento de UPP. El seguimiento de los pacientes se realizó entre diciembre de 2008 y marzo de 2009 en el hospital Universitario de Fuenlabrada.

Se ha calculado la frecuencia absoluta y relativa de UPP en los pacientes seguidos, la asociación entre variables cualitativas y la validez del registro en la HCE con respecto al patrón de referencia, el estudio observacional.

Resultados. Se ha completado un seguimiento a 1.001 pacientes encontrando 42 pacientes con UPP frente a los 25 pacientes que tenían registrada UPP en la HCE, siendo estadísticamente significativa la diferencia $(\mathrm{p}<0,001)$. El registro de la UPP en la HCE presenta un índice Kappa de 0,584 con una sensibilidad de $47,62 \%$ y una especificidad de $99,48 \%$ con respecto a la observación directa.

Conclusión. El número de pacientes que presentan UPP prácticamente se duplica si la recogida de datos es observacional por una enfermera experta frente a lo registrado en la HCE.

Palabras clave. Úlcera por presión. Epidemiología. Validez de las pruebas. Hospital. Historia clínica electrónica.

\begin{abstract}
Background. To compare pressure ulcers (PU) found in this prospective observational study with $\mathrm{PU}$ recorded in the Electronic Medical Record (EHR).

Method. We use a prospective observational study to record PU, with daily monitoring by an observer in the medical-surgical units of hospitalization, as well as a clinical record review of PU tracking. Patient monitoring was conducted between December 2008 and March 2009 at Fuenlabrada University hospital.

We calculated the absolute and relative frequency of PU in the patients followed, as well as the association among qualitative variables and the validity of the registration in the EHR with regard to the reference standard, the observational study.
\end{abstract}

Results. Among the 1,001 patients followed-up in this study, 42 of them showed PU (4.2\% of the total patients), compared to $25(2.5 \%)$ patients who had registered PU in the Electronic Health Record, which means a statistically significant difference $(\mathrm{p}<0.001)$. The record of PU presents a Kappa index of 0.548 , with a sensitivity of $47.62 \%$ and a specificity of $99.48 \%$, with regard to the reference standard, the observational study.

Conclusion. The number of patients with pressure ulcers almost doubled if the observational data collection is done by an expert nurse compared to what was recorded in the EHR.

Key words. Pressure ulcer. Epidemiology. Validity. Hospital. Electronic health records.
1. Innovación y Desarrollo. Hospital de Fuenlabrada. Madrid

2. Hospitalización Médica. Hospital de Fuenlabrada. Madrid

3. Reanimación Postquirúrgica. Hospital de Fuenlabrada. Madrid

4. Universidad Rey Juan Carlos. Alcorcón. Madrid

* Addendum autores: C.G. Nogueiras Quintas, G. Martín Merino, M.R. García Martín, E. Velayos-Rodríguez, P. Serrano Balazote, R. Navalon Cebrían, L. Lechuga Suárez

Recepción: 11 de abril de 2013

Aceptación provisional: 24 de junio de 2013

Aceptación definitiva: 15 de julio de 2013

\section{Correspondencia:}

Tomás Sebastián Viana

Responsable de Área de Innovación y

Conocimiento

Camino del Molino, 2

28942 Fuenlabrada. Madrid

Email: tsebastian.hflr@salud.madrid.org

Este trabajo ha contado con la financiación de dos proyectos: proyecto $n^{2}$ PI070873, subvencionado por el Instituto de Salud Carlos III durante el año 2007 y el proyecto: "Implantación de prácticas seguras para la prevención de úlceras por presión" (Ministerio de Sanidad y Consumo y Comunidad de Madrid). BOE $\mathrm{n}^{\circ} 10$ de 12 de enero de 2009. 


\section{INTRODUCCIÓN}

Las úlceras por presión (UPP) constituyen un problema relevante en los pacientes hospitalizados en nuestro país ${ }^{1}$. Además la mayoría se consideran evitables ${ }^{2}$ y constituyen un importante problema económi$\mathrm{co}^{3}$.

En la actualidad son consideradas un problema de seguridad de paciente, recogido en los principales modelos de evaluación de la seguridad como indicador a evaluar; en ellos se recoge tanto la existencia de protocolos de evaluación de riesgo, su prevención y tratamiento, como la aplicación de éstos y de las medidas preventivas, así como la frecuencia en la que los pacientes se ulceran ${ }^{4,5}$. En uno de esos estudios, proyecto SENECA ${ }^{6}$ en una muestra de 33 hospitales en los que se revisaron 150 historias clínicas en cada uno de ellos (50 de pacientes de Cuidados Intensivos, 50 de pacientes médicos y 50 de pacientes del ámbito quirúrgico) se encontró que un $8,6 \%$ de los pacientes analizados presentaba UPP, aunque las frecuencias encontradas en los diferentes hospitales variaba sustancialmente con una amplitud de más de 20 puntos porcentuales entre ellos.

Así mismo la tasa de UPP por 1000 altas está recogida como uno de los indicadores clave del Sistema Nacional de Salud español y se mide con periodicidad anual a partir de los datos recogidos en el Conjunto Mínimo Básico de Datos (CMBD), presentando tasas de 16,25 por mil en Madrid ${ }^{7} \mathrm{y}$ tasas de 11,40 por mil a nivel nacional en el año $2009^{8}$. Si bien en la definición del indicador se reconoce como limitación del indicador la influencia de la exhaustividad del $\mathrm{CMBD}^{9}$.

Esta limitación se ha puesto de manifiesto en algunos estudios como realizado en el hospital Santiago Apóstol de Vitoria ${ }^{10}$ en el que se evidenció que el CMBD solo recogía el $25 \%$ de las úlceras declaradas por los profesionales de enfermería, o un estudio previo realizado en el hospital de Fuenlabrada en el que se constató que no se recogía en el CMBD la información relacionada con la enfermería (incluidas las UPP) y que cuando ésta se recogía de ma- nera selectiva se producían cambios en la codificación y en el peso medio del hospi$\mathrm{tal}^{11}$.

Por otra parte hay estudios en los que parece que se pone de manifiesto que los registros clínicos no recogen la realidad clínica de los pacientes, así nos encontramos con el estudio de Admensen y Tewes ${ }^{12}$ que recogía información de pacientes, de los registros y de las enfermeras que los atendían con diferentes técnicas (entrevista estructurada, grupos focales y análisis de documentación) y mediante la triangulación de técnicas se encontraron que solo un $31 \%$ de los problemas de los pacientes conocidos por las enfermeras se reflejaron en los registros y un dato más preocupante es que un tercio de los problemas de los pacientes no eran conocidos por las enfermeras.

Hay que destacar en este aspecto una revisión publicada en $2011^{13}$ que incluía 77 estudios de 15 países y analizaba los registros clínicos de enfermería. En ellos se veía que la concordancia entre el registro clínico y lo que les ocurría a los pacientes (con estudios observacionales, entrevistas a pacientes y enfermeras y evaluación del desempeño profesional) era baja, siendo una de las implicaciones para la práctica y una recomendación realizada en la revisión sobre la que trabajar y mejorar en la práctica clínica ${ }^{13}$.

En este mismo aspecto, un estudio australiano en el que revisaron las alergias recogidas en los registros clínicos y las reacciones alérgicas notificadas encontraron que en un $35 \%$ de los casos no había registrada ninguna alergia y que en el $16 \%$ de los casos se había registrado como desconocido ${ }^{14}$.

En nuestro entorno no hemos encontrado estudios que evalúen la calidad de la información de los registros clínicos de enfermería con respecto a la realidad clínica, aunque sí es de destacar el estudio de Matilde Celma ${ }^{15}$ que recogía el escaso interés de la enfermería en el registro de la actividad propia y del poco interés que tienen en general los profesionales de enfermería para las actividades autónomas.

Por otra parte, estudios previos realizados por parte de este equipo investigador, 
en los que se analizaban a partir de los registros clínicos el porcentaje de pacientes incontinentes y las dependencias para los autocuidados de los pacientes y su evolución durante el ingreso, encontraron problemas del registro en la historia clínica por parte del personal de enfermería. Resultó llamativo que no pudieran incluir en los cálculos más que el $50-55 \%$ de la población reclutada para el estudio, por falta de cumplimentación de los registros pese a que la información era relevante para la dispensación de cuidados durante el ingreso y la continuidad de cuidados al alta ${ }^{16}$.

Dada la importancia que tiene la UPP como indicador clave en la seguridad del paciente y como indicador relevante de los cuidados que se dispensan, así como de los problemas de la calidad de los registros clínicos, se ha planteado la necesidad de realizar un estudio para evaluar la conformidad o fiabilidad intermétodos (concordancia), y la sensibilidad y especificidad de los registros de la historia clínica, con respecto a la observación directa de un observador externo.

\section{MÉTODOS}

Se ha realizado un estudio de validez de pruebas diagnósticas, observacional y prospectivo, siguiendo a una serie de pacientes durante su ingreso en las unidades de hospitalización médico-quirúrgicas del hospital, a los que por una parte se les ha realizado una observación directa durante su estancia hospitalaria y por otra parte se ha revisado si tenían notificada alguna UPP en el registro clínico específico de la historia clínica electrónica (HCE) durante el periodo de estudio. El seguimiento se realizó de diciembre de 2008 a marzo de 2009 y fue autorizado por la Dirección del Centro y evaluado por la Comisión de Investigación del hospital y se enmarcó en un proyecto de evaluación de calidad de registros para el seguimiento de la implantación de prácticas seguras en el manejo de las UPP. A los pacientes, de forma previa a la observación directa, se le informó del objeto del estudio y se les solicitó consentimiento para recoger la información y hacer la exploración.
El estudio se ha realizado en el hospital Universitario de Fuenlabrada. Éste es un hospital de nivel 2 con 406 camas instaladas, que en el año 2009 tuvo un promedio de 289,4 camas funcionantes y 15.592 altas hospitalarias $^{17}$.

Para realizar el estudio, se estimó un tamaño muestral de 951 pacientes, considerando una prevalencia de UPP de un $2 \%$ (prevalencia registrada en el histórico del hospital que al ser más baja que la encontrada en la literatura fue la que se escogió porque hacía necesario un tamaño de muestra mayor); una sensibilidad del registro frente al patrón de referencia (observación diaria por un observador externo) del $95 \%$, dado que al no evaluar coincidencia en grado, localización..., se esperaba que la totalidad de las UPP que se pudieran encontrar en la observación directa estuvieran registradas en el registro específico de la HCE, un nivel de confianza del 95\% y una precisión relativa del $10 \%$.

Para realizar la observación directa de los pacientes, se seleccionó a una enfermera asistencial, miembro del grupo de UPP del hospital, que durante el estudio visitaba los días laborables a los pacientes ingresados en las unidades de estudio. En la visita les preguntaba y revisaba con el paciente, si desde su anterior visita habían tenido algún tipo de incidente, registrando en una base de datos elaborado ad-hoc para el estudio si presentaba UPP La observadora no accedía a la historia clínica y tenía instrucciones de no comentar las situaciones clínicas de los pacientes con el personal de la unidad.

Por otra parte, a estos mismos pacientes que se les había hecho la observación directa, se les realizó una extracción automatizada de la información contenida en el registro clínico especifico de la UPP en la HCE. Con ello se pretendía verificar si el personal de la unidad había registrado alguna UPP, con el objeto de evaluar la fiabilidad intermétodos o de conformidad y la validez del registro.

Los resultados se presentan para las variables cualitativas con su distribución de frecuencias y para las cuantitativas con su media y desviación estándar. 
Se han calculado las frecuencias absolutas y relativas de UPP, tanto según la información obtenida con la observación directa, como con la obtenida a través del registro de UPP del hospital.

Se ha medido la sensibilidad, especificidad, valor predictivo positivo y valor predictivo negativo del sistema de notificación de UPP con respecto al patrón de referencia de la observación diaria de los pacientes por un observador externo, que se ha considerado el patrón de referencia.

Dadas las limitaciones que puede presentar el patrón de referencia, ya que éste era también una enfermera asistencial, cuya única diferencia con el resto de enfermeras que asistían a los pacientes e iban a registrar en la $\mathrm{HCE}$, era que pertenecía al grupo de trabajo de UPP y que durante el estudio solo se dedicó a hacer la observación directa, se ha evaluado también la fiabilidad intermétodos ${ }^{(18)}$ o conformi$\operatorname{dad}^{(19)}$, entre el registro de UPP y las UPP encontradas con el patrón de referencia (la observación directa) mediante el índice de concordancia Kappa, y se ha calculado la diferencia de proporciones para muestras emparejadas, que se ha contrastado con el test de McNemar(18).

En todos los contrastes de hipótesis se ha rechazado la hipótesis nula con un error de tipo I o $\alpha$ menor a 0,05 y los resultados se presentan con sus intervalos de confianza al $95 \%$.

\section{RESULTADOS}

Se ha completado un seguimiento prospectivo a 1.001 pacientes en el periodo de estudio, habiendo realizado un total de 4.486 observaciones, con una media de 4,5 observaciones por paciente.

Las características sociodemográficas de los pacientes global y clasificada en pacientes con UPP y sin UPP, pueden verse en la tabla 1.

Tabla 1. Características socio-demográficas

\begin{tabular}{lcccc}
\hline & $\begin{array}{c}\text { Pacientes con UPP } \\
\text { Media (IC 95\%) } \\
\mathbf{n = ~ 4 2}\end{array}$ & $\begin{array}{c}\text { Pacientes sin UPP } \\
\text { Media (IC 95\%) } \\
\mathbf{n = ~ 9 5 9}\end{array}$ & $\begin{array}{c}\text { Total pacientes } \\
\text { Media (IC 95\%) } \\
\mathbf{n = 1 . 0 0 1}\end{array}$ \\
\hline Edad & $78,9(74,9-82,9)$ & $62,8(61,6-64,1)$ & $63,5(62,3-64,7)$ \\
\hline Estancia & & $21,64(14,7-28,6)$ & $9,2(8,5-9,8)$ & $9,7(9-10,4)$ \\
\hline Sexo* & Hombre & $19(45,2)$ & $477(49,7)$ & $496(49,6)$ \\
Sexo* $^{*}$ & Mujer & $23(54,8)$ & $482(50,3)$ & $505(50,4)$ \\
\hline
\end{tabular}

${ }^{*} \mathrm{~N}(\%)$.

Se encontraron en la observación directa por la observadora externa un total de 42 pacientes que presentaron una o varias UPP. Esto supone que un $4,2 \%$ de los pacientes seguidos presentaron UPP, con un intervalo de confianza al $95 \%$ de $(2,9-5,5)$. El número total de úlceras era de 70 . Presentaron una úlcera, 24 pacientes $(57,1 \%)$; 2 úlceras, 10 pacientes (23,8\%); 3 úlceras, 7 pacientes $(16,7 \%)$ y 5 úlceras, un paciente (2,4\%).

Las localizaciones más frecuentes han sido: talones 28 úlceras $(40,0 \%)$; sacro- glúteo $24(34,3 \%)$; espalda $5(7,1 \%)$; maléolos 5 (7,1\%); trocánteres 3 (4,3\%); codos 2 (2,9\%); rodillas $2(2,9 \%)$; pene $1(1,4 \%)$.

Los estadios de presentación de las úlceras no se han podido registrar más que en 45 UPP ya que la observadora, ni levantaba las curas, ni revisaba las historias clínicas. Los estadios registrados han sido: estadio 1:24 pacientes (53,3\%); estadio $2: 20$ pacientes $(44,4 \%)$; estadio $3: 1$ paciente $(2,2 \%)$.

De los pacientes seguidos, tenían notificado en el formulario especifico de UPP 
25 pacientes $(2,5 \%)$, con un intervalo de confianza al 95\% (1,5-3,5). Coincidían con la observación directa 20 pacientes y de los 22 restantes, en 8 pacientes aunque en el registro de UPP no estaba recogido, sí que aparecían en las observaciones de enfermería comentarios del tipo "enrojecimiento" "flictena", "zona enrojecida". Descontando estos pacientes, en total hubo 14 $(33,3 \%)$ que en el seguimiento por la obser- vadora externa se detectó y registró que tenían una UPP y en los registros clínicos y observaciones no había ninguna mención, ni se había registrado UPP como tal en el formulario específico.

La diferencia de proporciones de UPP encontrada entre el registro de UPP y el porcentaje de UPP encontrado en el estudio observacional ha sido de $-1,698$ con una p de 0,002 (Tabla 2).

Tabla 2. Análisis detección y registro de úlceras por presión

\begin{tabular}{lclcc}
\hline & $\begin{array}{c}\text { Pacientes con úlcera } \\
\mathbf{N}(\%)\end{array}$ & $\begin{array}{c}\text { Diferencia de } \\
\text { proporciones } \\
\text { Dif. (IC 95\%) }\end{array}$ & $\begin{array}{c}\text { Test } \\
\text { McNemar }\end{array}$ & p \\
\hline UPP observación directa & $42(4,2)$ & $-1,698(-2,710 ;-0,686)$ & 9,4815 & 0,0021 \\
\hline UPP registrada en HCE & $25(2,5)$ & & & \\
\hline
\end{tabular}

La fiabilidad intermétodos o conformidad entre las dos observaciones se ha calculado con el índice Kappa que ha sido de 0,584 , con un intervalo de confianza del $95 \%$ de $(0,442-0,726)$, lo cual debe ser interpretado como una concordancia moderada según la escala de interpretación propuesta por Altman ${ }^{20}$.

La validez del registro con respecto a la observación directa presenta una sensibilidad del registro específico de UPP del $47,62 \%$ con un IC de 31,32-63,91 y una precisión relativa final de la muestra de $15,1 \%$; una especificidad del 99,48\% $(98,97-99,99)$ y una precisión relativa final de la muestra de $0,5 \%$; un valor predictivo positivo del $80 \%(63,32-97,68)$ y un valor predictivo negativo del 97,75\% 96,76-98,73).

\section{DISCUSIÓN}

En este estudio se ha observado que la frecuencia de pacientes con UPP prácticamente se duplica si la recogida de datos se hace de forma observacional frente al sistema de registro clínico y de notificación que tiene implantado el hospital. Es de destacar que en el estudio se ha encon- trado una sensibilidad baja, al quedar más de la mitad de los pacientes con UPP sin registrar en el registro clínico específico, aunque cuenta con un valor predictivo positivo aceptable, es decir que los pacientes que tienen registrada la UPP realmente la presentan. La especificidad y el valor predictivo negativo son muy buenos, aunque esto tiene menos trascendencia de cara al objetivo del estudio.

La repercusión clínica es claramente relevante, ya que el registro clínico es lo que utiliza el profesional para registrar los problemas y acontecimientos que le suceden el paciente y para hacer el seguimiento y planificación de los cuidados. Sorprende en el estudio la baja sensibilidad que ha tenido el registro clínico que hace necesario estudiar con nuevas estrategias y con detalle este hallazgo.

Si bien es cierto que no se han encontrado estudios similares en nuestro medio para saber si éste es un hecho anecdótico de nuestro hospital o bien una realidad en los hospitales de nuestro entorno, lo cierto es que la literatura recoge estudios en los que se constata la baja concordancia del registro en la historia clínica con respecto 
a la realidad clínica ${ }^{12,13}$, así como con la planificación de los cuidados de los pacientes en $\mathrm{UPP}^{21}$, con problemas como el registro en la historia clínica de las caídas ${ }^{22,23}$, o con las alergias a medicamentos ${ }^{14}$ por ello no es descartable que esta misma situación en la que el número de úlceras registradas en la HCE es netamente inferior al de UPP que presentan los pacientes, sea algo generalizado en el resto de hospitales. En todo caso con los datos encontrados en este estudio parece que el registro clínico de las UPP no es el método idóneo para la notificación y el conocimiento de las UPP que aparecen en las unidades asistenciales, dada la baja sensibilidad y la concordancia moderada encontrada.

El problema tiene gran trascendencia dado que el caso de las UPP es un indicador clave del Sistema Nacional de Salud ${ }^{9}$ y su aparición un indicador de seguridad de paciente reconocido a nivel nacional e internacional ${ }^{4,5,24}$, hecho aun más preocupante si se tiene en cuenta que hay estudios que ponen de manifiesto que solo un $25 \%$ de las UPP ${ }^{10}$ conocidas se recogen en el Conjunto Mínimo Básico de Datos que es la fuente fundamental de la que se nutren estos indicadores

Cuantificar el problema y unificar y estandarizar la forma de medirlo parece relevante a la vista de los datos encontrados. Se deberían realizar estudios de mayor amplitud que recojan las limitaciones que tienen las diferentes maneras de abordar el problema y permitan evaluar mejor los cuidados que se dispensan y el conocimiento de los pacientes que realmente presentan UPP.

Resulta preocupante que en un tema en el que la enfermería es tan sensible se hayan encontrado estas carencias en el registro clínico. Esto abre las puertas a la investigación sobre lo que estará ocurriendo con otras realidades clínicas, el grado de generalización de este problema y las causas que pueden estar generándolo ${ }^{15}$. En todo caso, es de destacar la necesidad de revisar la calidad de los registros clínicos con los que se está trabajando, ya que como apunta Yocum ${ }^{25}$ "la práctica enfermera y los estándares profesionales requieren registrar y los registros son necesarios para los pacientes" de ahí que uno de los aspectos fundamentales de cualquier práctica clínica de calidad y que busque la seguridad será contar con unos sistemas de registro clínico efectivos que den soporte a la decisión y a la atención a los pacientes y con los resultados encontrados en este estudio y en la bibliografía parece que la calidad de los registros presenta limitaciones importantes.

A la hora de considerar los resultados, aunque el estudio ha sido prospectivo, hay que tener en cuenta que la observadora externa ha sido una enfermera experta en UPP, lo que ha podido condicionar que la detección de UPP de grado I, las más difíciles de diagnosticar y detectar, se hayan sobrediagnosticado en este estudio con respecto a lo que se hace habitualmente. El número de pacientes con observaciones en los comentarios en texto libre en las HCE encontradas y que no tenían registrada UPP parece apuntar a ello. Un porcentaje muy elevado de pacientes no tenían registrada en la HCE ningún tipo de información sobre las UPP que padecían, ni en los registros estructurados, ni en el texto libre.

Por otro lado, no se ha podido enmascarar la observación directa, si bien la observadora tenía instrucciones de no revisar las HCE y de no comentar información clínica con el personal de la unidad, por lo que este hecho ha podido condicionar la cantidad y/o calidad del registro de los profesionales.

Como conclusión del estudio se puede decir que éste apunta a que la calidad de los registros clínicos específicos de UPP para conocer los pacientes que tienen UPP con respecto a una observación directa es bastante limitada. Por ello, habría que interpretar con cautela la información existente sobre las UPP en hospitales de agudos y que, no solo es importante conocer la frecuencia de UPP, sino también el método que se ha utilizado para evaluarlas. Además dadas las diferencias y las limitaciones en los datos entre las diferentes formas de medirlo, habría que considerar que el problema es de mayor magnitud que lo que las cifras publicadas reflejan. 


\section{BIBLIOGRAFÍA}

1. Soldevilla Agreda JJ, Torra i Bou JE, Verdú Soriano J, López Casanova P. $3^{\text {er }}$ Estudio de Prevalencia de Úlceras por Presión en España, 2009. Epidemiología y variables definitorias de las lesiones y pacientes. Gerokomos 2011; $22: 77-90$.

2. JoAnna BRiggs. Institute of Evidence Based Nursing and Midwifery. Lesiones por presión-prevención de las lesiones por presión. Best Practice 2008; 12 (2).

3. Soldevilla Agreda J, Torra i Bou J, Posnett J, et al. Una aproximación al impacto del coste económico del tratamiento de las úlceras por presión en España. Gerokomos 2007; 18: 201-210.

4. Agencia de Calidad del Sistema Nacional de Salud. Construcción y validación de indicadores de buenas prácticas sobre seguridad del paciente. $1^{\mathrm{a}}$ ed. Madrid: Ministerio de Sanidad y Consumo; 2008.

5. Secretaria General Técnica MSPS. Estándares de calidad de cuidados para la seguridad del paciente en los hospitales del SNS. Proyecto Séneca. $1^{\mathrm{a}}$ ed. Madrid: Ministerio de Sanidad y Política Social; 2009.

6. Agencia de Calidad del Sistema Nacional de Salud. La seguridad en los cuidados del paciente hospitalizado. Proyecto SENECA. $1^{\underline{a}}$ ed. Madrid: Ministerio Sanidad y Política Social; 2010.

7. Ministerio Sanidad, Servicios Sociales e Igualdad. Indicadores clave del Sistema Nacional de Salud. Madrid. 2012; [consultado 10-03-2012]: Disponible en: http://www.msps. es/estadEstudios/estadisticas/sisInfSanSNS/ docsInclaSNS2011/13_INCLASNS-MADRID. pdf.

8. Ministerio Sanidad, Servicios Sociales e Igualdad. Indicadores clave del Sistema Nacional de Salud. España. 2011; [consultado 10-03-2012]: Disponible en: http://www.msps. es/estadEstudios/estadisticas/sisInfSanSNS/ docsInclaSNS2011/00_INCLASNS-ESPANNA. pdf.

9. Ministerio Sanidad, Servicios Sociales e Igualdad. Sistema de información del Sistema Nacional de Salud. Indicadores clave 2013; [consultado 10-03-2012]: Disponible en: http://www.msps.es/estadEstudios/ estadisticas/sisInfSanSNS/docsInclaSNS/INCLASNS_2012_MetodyFichas_.pdf.

10. Cidoncha Moreno Ma, Campo Martínez C, Gamarra-Mayor Martínez MT, Alonso Castillo J. Investigando la declaración de la úlcera por presión en la asignación de los grupos rela- cionados por el diagnóstico (GRD). Gestión hospitalaria 2003; 14: 94-98.

11. Sebastián Viana T, Del Pozo-Herranz P, Navalón Cebrián R, Lema Lorenzo I, Nogueiras Quintas CG. Impacto de la codificación de información de enfermería en el peso medio de los grupos relacionados con el diagnóstico y su repercusión en la facturación de un hospital. Gac Sanit 2009; 23: 55-57.

12. Adamsen L, Tewes M. Discrepancy between patients' perspectives, staff's documentation and reflections on basic nursing care. Scand J Caring Sci 2000; 14: 120-129.

13. Wang N, Halley D, Yu P. Quality of nursing documentation and approaches to its evaluation: a mixed-method systematic review. J Adv Nurs 2011; 67: 1858-1875.

14. Khalil H, Leversha A, Khalil V. Drug allergy documentation-time for a change? Int J Clin Pharm 2011; 33: 610-613.

15. Celma Vicente M. Género y cultura organizacional administradores del poder entre enfermeras y médicos. Tesela 2007;1. [consultado 10-03-2012]: Disponible en http://www. index-f.com/tesela/ts1/ts6329r.php

16. Sebastián-Viana T, Del Pozo-Herranz P, Gil-HerNÁNDEZ MR. Evolución de la incontinencia y nivel de autonomía en pacientes ingresados en el Hospital Fuenlabrada. Evidentia 2009 6(25) [consultado 10-03-2012]: Disponible en: http://www.index-f.com/evidentia/n25/ ev6859r.php

17. Hospital de Fuenlabrada. Memoria 2009. 2010 [consultado 10-03-2012]: Disponible en: http:// www.madrid.org/cs/Satellite?blobcol=urldata \&blobheader=application\%2Fpdf\&blobheade rname1=Content-disposition\&blobheaderna me $2=$ cadena\&blobheadervalue1 =filename\%3 DMEMORIA+Fuenlabrada. pdf\&blobheaderv alue2=language\%3Des\%26site\%3DPortalSalu d\&blobkey=id\&blobtable=MungoBlobs\&blob where $=1271650034062 \&$ ssbinary $=$ true.

18. SzkLo M, NiEto J. Epidemiología intermedia. Conceptos y aplicaciones. $1^{\mathrm{a}}$ ed. Madrid: Díaz de Santos; 2003.

19. Martín Andrés A, Luna del Castillo JD. Medidas de concordancia o acuerdo. Bioestadística para las ciencias de la salud. $1^{\mathrm{a}}$ ed. Madrid: Norma-Capitel; 2004; 389-401.

20. Altman D. Practical statistics for medical research. New York: Chapman and Hall; 1991.

21. O BRIEn JA, Cowman S. An exploration of nursing documentation of pressure ulcer care in an acute setting in Ireland. J Wound Care 2011; 20: 197-203.

22. Sebastián Viana T, García Martín MR, NúÑez Crespo F, Velayos Rodríguez EM, Martín Merino 
G, GonZÁlez Ruz JM et al. ¿Cuál es la incidencia de caídas real en un hospital? Enferm Clínica 2011; 21: 271-274.

23. Hill-Westmoreland EE, Gruber-Baldin AL. Falls documentation in nursing homes: agreement between the minimum data set and chart abstractions of medical and nursing docu- mentation. J Am Geriatr Soc 2005; 53: 268273.

24. Aranaz J, Aibar C, Vitaller C, Ruiz R. Estudio Nacional de Efectos Adversos ligados a la hospitalización. Estudio ENEAS. 2006.

25. Yocum F. Registros de enfermería: Cuidados de calidad. Nursing (Ed Esp ) 2003; 21: 22-27. 